\title{
Smart illuminative Charging (SiC) of Future Electric Vehicles Using Roadway Infrastructure
}

\author{
Daniel Fernandez ${ }^{1}$, Ann Sebastian ${ }^{1}$, Ethan $\mathrm{Ahn}^{1}$, Mahmoud Reda Taha ${ }^{2}$, Samer Dessouky ${ }^{3}$, and Sara Ahmed ${ }^{1, *}$ \\ ${ }^{1}$ Department of Electrical and Computer Engineering, The University of Texas at San Antonio, San Antonio, TX 78249 \\ ${ }^{2}$ Department of Civil, Construction and Environmental Engineering, University of New Mexico, Albuquerque, NM 87131-1070 \\ ${ }^{3}$ Department of Civil and Environmental Engineering, The University of Texas at San Antonio, San Antonio, TX 78249
}

\begin{abstract}
Inspired by the fact that there is an immense amount of renewable energy sources available on the roadways such as mechanical pressure and frictional heat, this paper presents the development and implementation of an innovative charging technique for future electric vehicles (EVs) by fully utilizing the existing roadways and the state-of-the-art nanotechnology. The paper introduces a novel wireless charging system that uses LEDs powered by piezoelectric nanomaterials as the energy transmitter source and thin film solar panels placed at the bottom of the EVs as the receiver, which is then poised to deliver the harvested energy to the vehicle's battery. The lab-scale prototype device was developed to testify the proposed mechanism of illuminative charging (i.e., "light" couples pavement and vehicle as a wireless energy transfer medium), and the synthesized large-area nanomaterial produced high output voltages of up to $52 \mathrm{mV}$ upon mechanical pressure.
\end{abstract}

\section{Introduction}

Roadways represent an important part of transportation infrastructure, serving as a backbone to enable better mobility for people and goods. Because roadways greatly impact the economic growth and development of communities, state-of-the-art research has focused on planning, designing, and constructing roadways to reduce environmental impact, increase sustainability, or improve the efficiency of transportation flow. However, due to increasingly large demands for reduced driver stress, independent mobility for nondrivers, and increased safety and in-vehicle infotainment, further research is called for on the development of novel roadways that can best adapt for future vehicles. For example, electric vehicles (EVs) are promoted as a key contributor to building a more sustainable mobility system as they are generally much more energy efficient than those powered by fossil fuels. Furthermore, increasing the use of these electric cars will result in a considerably lower emission of carbon dioxide and other air pollutants, including nitrogen oxides and particulate matter (PM), thereby improving air quality and preserving the environment. However, an existing challenge is that additional electricity is required to charge EV batteries, which can significantly offset EV's benefits through additional emissions resulting from increased electricity production. In addition, modern EVs still need to be equipped with a large-capacity battery pack as an energy storage unit to operate for a satisfactory distance. Lithium-ion batteries, which are the most commonly used, have a specific energy density of only $90-100 \mathrm{Wh} / \mathrm{kg}[1,2]$, which is very low as compared with that of gasoline (about $12,000 \mathrm{Wh} / \mathrm{kg}$ ). Therefore, the lithium-ion battery operated EVs can only travel for about 300 miles until they need to be recharged. In addition to the low energy density, contemporary battery technologies have shortcomings of long charging time, large size and weight, limited life time, and relatively high cost. To overcome these challenges, this paper presents an alternative charging strategy that harvests renewable energy sources available on the roadways by using the piezoelectric nanomaterials.

\section{Results}

The smart illuminative charging ( $\mathrm{SiC}$ ) system developed in this work features the following three pathways of energy conversion; (1) the mechanical energies available on the roadway are converted into electrical energies through the use of energy-harvestable 2D nanomaterials $(\mathrm{EH} 2 \mathrm{~N}),(2)$ these harvested energies are used (instead of the conventional power grid) to power the LEDs embedded in the roadway, and (3) the PV panels attached to the vehicle bottom finally convert the received light energy back to electricity (See Figure 1 for the schematic of $\mathrm{SiC}$ ).

\footnotetext{
* Corresponding author: sara.ahmed@utsa.edu
} 


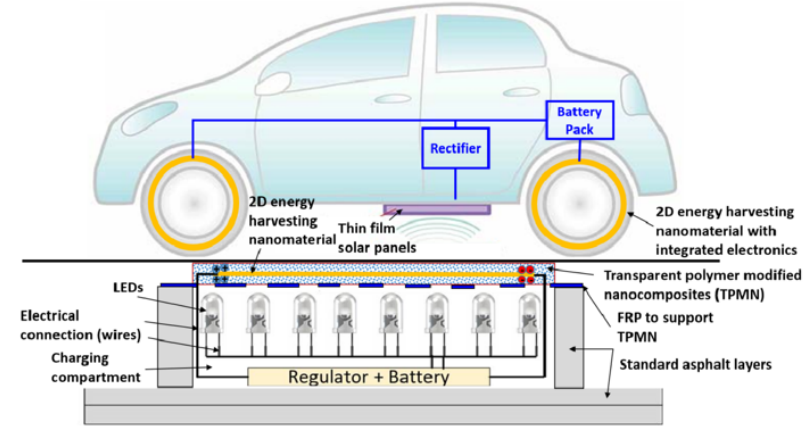

Fig. 1. Schematic of the smart illuminative charging ( $\mathrm{SiC})$ system proposed for vehicles and roadway infrastructures of the future.

\subsection{Energy-harvestable 2D nanomaterials (EH2N)}

Two-dimensional (2D) nanomaterials [3] are an emerging class of materials with properties making them highly attractive for both fundamental exploration of physical phenomena and practical engineering applications across a variety of disciplines including energy harvesting. Accordingly, the past years have experienced a strong resurgence of interest in 2D nanomaterials (i.e., beyond graphene), sparked by the demonstration of the first transistor based on them [4] and the discovery of strong photoluminescence in atomically-thin monolayers of them [5]. These 2D nanomaterials exhibit a unique, unmatched portfolio of functionalities and capabilities beyond the conventional materials at the fundamental scaling limit (thicknesses of a few nanometers), and favorable electronic, mechanical, and optical properties. For the purpose of this work, their promising piezoelectric (energy harvesting) properties with excellent flexibility and transparency are of particular interest. One key challenge, however, in utilizing these energy-harvestable 2D nanomaterials (EH2Ns) for the development of practical electromechanical systems has been controllable growth or synthesis of high-quality 2D nanomaterials [6]. Unlike most of the previous works [7, 8], this work features the chemical vapor deposition (CVD)-based large-area material preparation, because the area of otherwise "exfoliated" 2D nanomaterial flakes is typically limited to hundreds of micrometers squared, thus limiting their adoption in many practical applications that require large-area integration such as in the proposed $\mathrm{SiC}$ system for electric vehicles. The CVD process is more scalable in the sense that growth is only limited by the CVD system itself. In this paper, the high-quality, large-area (up to $2 \mathrm{~cm} \times 2 \mathrm{~cm}$ ) EH2N was prepared and characterized for the novel purpose of using them as a scalable, mechanically robust energy harvesting source that can be integrated in the existing roadway infrastructure.

\subsubsection{Sample Preparation}

Figure 2 shows the 2D nanomaterials prepared by the CVD technique (in collaboration with researchers at
University of Central Florida [9]). The two " $2 \mathrm{~cm} \times 2$ $\mathrm{cm}$ " square samples in the figure represent the energyharvestable 2D nanomaterials $(\mathrm{EH} 2 \mathrm{~N})$ successfully synthesized and deposited on the $\mathrm{SiO}_{2} / \mathrm{Si}$ substrate in the quartz tube CVD furnace at a relatively large scale using the method described in the literature [9]. The thicknesses of EH2N range from $1.5 \mathrm{~nm}$ to $2 \mathrm{~nm}$ as confirmed in the cross-sectional transmission electron micrograph (TEM) image shown in the inset. It is highlighted that a commercially available substrate material $\left(\mathrm{SiO}_{2} / \mathrm{Si}\right)$ was used for nanomaterial synthesis and the nanomaterial can be readily transferred to any other substrate. This will facilitate a subsequent device or system integration process.

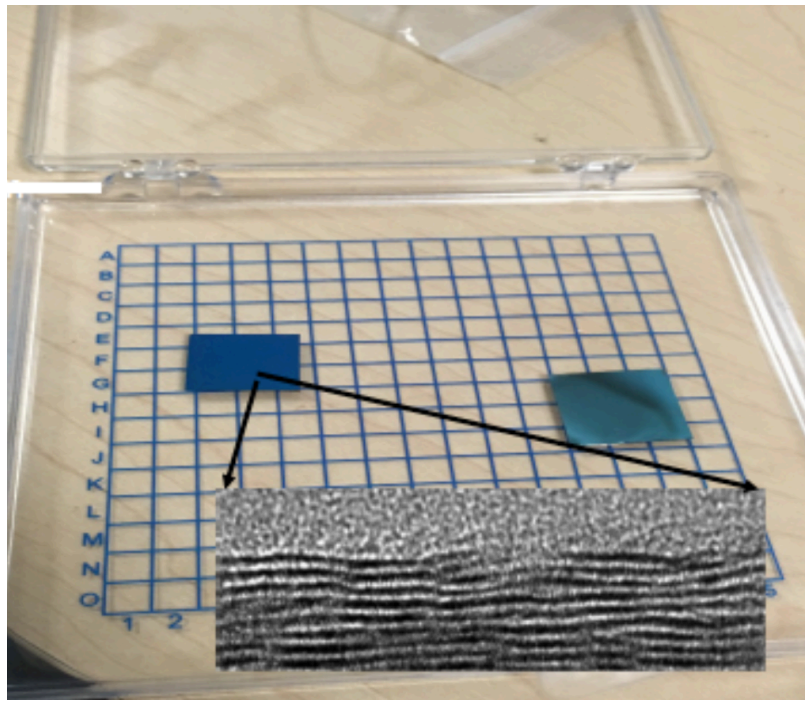

Fig. 2. EH2N grown by CVD.

\subsubsection{Structural characterization}

The prepared EH2N material was studied using the Raman Spectroscopy technique, which is a wellestablished tool used to analyze a variety of lowdimensional nanomaterials, including graphene, carbon nanotubes, and transition metal dichalcogenides [10]. Figure 3 shows the measured Raman spectrum of asgrown $\mathrm{EH} 2 \mathrm{~N}$ layers on a $\mathrm{SiO}_{2} / \mathrm{Si}$ substrate, indicating two distinct peaks corresponding to the in-plane (E12g) and out-of-plane (A1g) lattice vibration modes. Other characterization methodologies such as AFM (atomic force microscope), EDS (energy-dispersive x-ray spectroscope), and SEM (scanning electron microscope) were also applied to further investigate the structural integrity of the prepared EH2N samples (although not shown). This set of structural characterizations confirms that the EH2N thin film was prepared with no significant amounts of crystalline defects and imperfections, which is critical to ensure its piezoelectric (energy harvesting) behavior.

\subsubsection{Piezoelectric characterization}

In this work, the EH2N's piezoelectricity was experimentally characterized by adopting the 
piezoresponse force microcopy (PFM) technique (Figure 4). The PFM is a well-established approach for probing piezoelectric properties at the nanoscale, based on the coupling between polarization and mechanical displacement; a highly localized electric field is applied to the material and then the resultant surface displacement is measured. The following equations provide an elementary theory of the PFM measurement.

$$
\begin{gathered}
V_{t i p}=V_{d c}+V_{a c} \cos \omega t \\
A=A_{0}+A_{1} \omega \cos (\omega t+p)
\end{gathered}
$$

Here, $V_{t i p}$ is the voltage applied to the conductive tip, $V_{d c}$ is the dc voltage bias (switching bias), $V_{a c}$ is the ac voltage bias (probing bias), and $\omega$ is the driving frequency of the ac bias. As the sample expands or contracts due to the inverse piezoelectric effect, the tip deflection can be monitored using a lock-in amplifier because the tip oscillation (A) will have the phase shift (p) between the driving voltage $\left(V_{a c}\right)$ and the voltageinduced mechanical deformation $\left(A_{l \omega}\right)$ as well as the static surface displacement $\left(A_{0}\right)$. Figure 4 shows a decent piezoresponse signal exhibited by the EH2N. When the polarization and applied electric field were parallel (left subpanel, $+10 \mathrm{~V}$ applied), the deformation was positive (i.e., expansion) and the PFM signal was in phase with $V_{a c}$. On the contrary, when the opposite electric field ($10 \mathrm{~V}$ ) was applied, this caused the EH2N to contract with the consequent lowering of the PFM cantilever tip. This led to the $180^{\circ}$ phase shift as seen in the right subpanel. Given that all materials that exhibit this so-called inverse piezoelectric effect are essentially piezoelectric, this measurement proves that the prepared nanomaterial $(\mathrm{EH} 2 \mathrm{~N})$ is a strong piezoelectric material that can be harnessed for the energy harvesting or mechanical-toelectrical energy conversion purpose.

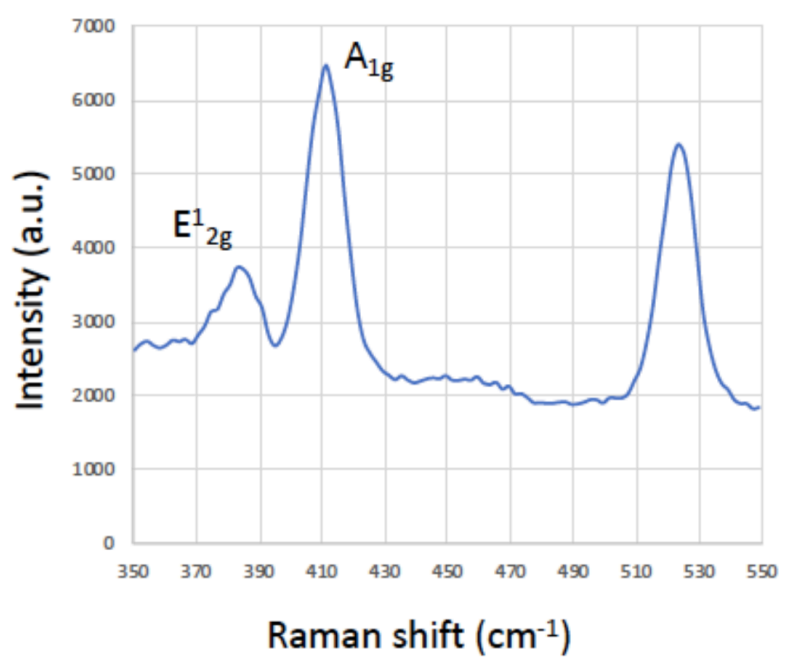

Fig. 3. Raman characterization data of $\mathrm{EH} 2 \mathrm{~N}$ grown on a $\mathrm{SiO}_{2} / \mathrm{Si}$ substrate.

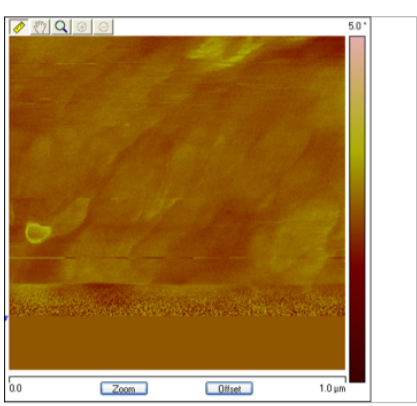

$+10 \mathrm{~V}$

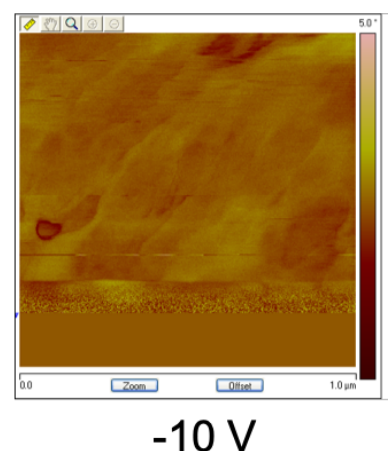

Fig. 4. PFM measurements of EH2N. The observed phase shift between the two different voltage polarities indicate that the EH2N thin film expands and contracts with the electric field direction (i.e., piezoelectric).

\subsubsection{Harvested voltage from $\mathrm{EH} 2 \mathrm{~N}$}

To further emulate the practical situation where the nanomaterial (embedded in roadways) is exposed to a significant amount of mechanical pressure, the EH2N sample's energy harvesting property was examined by bending it and directly measuring the induced output voltage (i.e., piezoelectrically induced polarization charges). For this experiment, the $\mathrm{EH} 2 \mathrm{~N}$ was transferred onto a flexible substrate (polyimide film) and the electrode was deposited on sides by the e-beam evaporation and shadow mask techniques. As seen in Figure 5 , the mechanically strained (by $0.5 \%$ to $1 \%$ ) nanomaterial was able to produce an output voltage of up to $52 \mathrm{mV}$, which is higher than the state-of-the-art reported in the literature [7]. This result proves the EH2N's potential to become an alternative energy source from the ground when embedded in roadways.

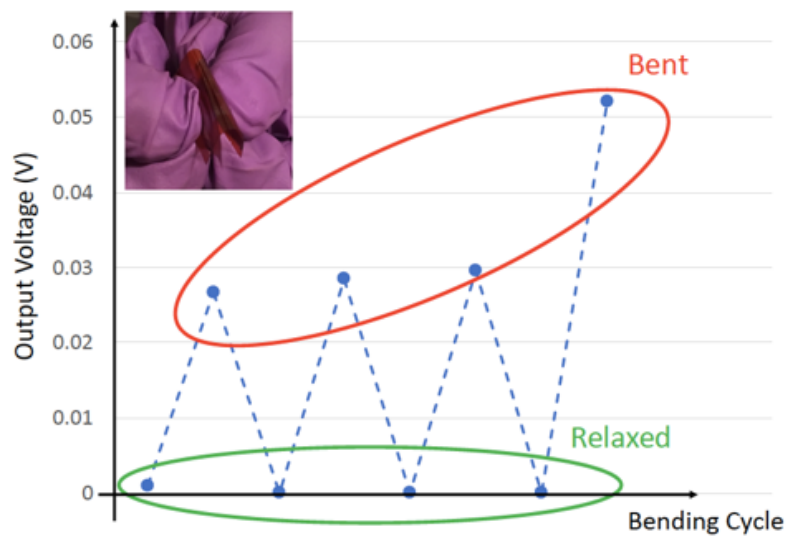

Fig. 5. Bending experiment and the produced output voltage.

\subsection{Light emitting diodes (LEDs) embedded in roadway}

The LEDs serve as the key element in transmitting the energy in the form of light in the proposed smart charging system and they should have both high energyconversion efficiency and be flexible because they will be powered by the nanomaterials embedded together in the roadway pavement. The energy efficiency in LEDs is 
typically measured in lumens per watt. The Samsung LT-Q562A LED strip was chosen in this work, as it emits light with relatively high energy efficiency (approximately 203 lumens per watt). The strips are also semi-flexible and therefore they fit the proposed application in this work.

\subsection{Solar panels attached in vehicle}

The solar (photovoltaic, PV) panels serve as the receiving end of the light-mediated energy transfer in the proposed $\mathrm{SiC}$ system. The solar panels available in the market are divided into three types, typically referred to as three generations. The first generation are the conventional (wafer-based) solar cells made of mono- or poly-crystalline silicon. The second generation of solar cells are known as thin-film ones, with the advantage of being flexible. The third generation indicates emerging PV technologies, which are still in the prototype phase and not commercially available.

The first generation of solar cells are still the most widely used for residential and commercial applications. The reasoning behind this is due to having the highest efficiency and life span. The drawback is the larger surface area and the higher manufacturing cost compared to the second generation of solar cells. The thin-film, second generation, solar panels are commonly made from amorphous silicon (a-Si), cadmium telluride (CdTe), or copper indium gallium selenide (CIGS) substrates. These solar cells have better characteristics for high temperatures and shading. The huge selling point of these solar cells are the flexibility, and their ability to contour to a surface.

For the $\mathrm{SiC}$ system in this work, two solar panels were utilized. One is a standard first-generation monocrystalline solar panel constructed of a tempered glass front, EVA (ethyl vinyl acetate) pottant, and a PVF (polyvinyl fluoride) backing to provide protection from environmental conditions. The entire laminate is framed in a heavy-duty anodized aluminum frame to provide structural strength. This solar panel has an output of 10 $W, 12 \mathrm{~V}$ and efficiency greater than $17 \%$. The other combines the first- and second-generation solar panels, thus being mono-crystalline and flexible. This hybrid solar panel features, using a transparent epoxy to protect the solar cells from heat and water, a rare combination of being light-weight, flexible and efficient, which has the output specification of $13 \mathrm{~W}$ and $5 \mathrm{~V}$ with a $23.5 \%$ efficiency

\subsection{Lab-scale prototype of SiC system}

Figure 6 shows the lab-scale proof-of-concept prototype of $\mathrm{SiC}$, designed and implemented in this work. The prototype consists of a 10' track with five 2' divisions (Figure 6 shows only one division); the divisions alternate from wood to plexiglass, starting and ending with wood. The LEDs are located under the transparent plexiglass which simulates the portion of roadway pavements with EH2Ns embedded. The $\mathrm{SiC}$ prototype had 16 strips of LEDs (each strip having 40 LEDs with rating of $21.9 \mathrm{~V}$ and $450 \mathrm{~mA}$ ), and these strips were connected in parallel to have a constant voltage. The LEDs were powered by a DC source in the prototype for the purpose of demonstration. Given that the piezoelectric voltage per area obtained from nanomaterials is about $12 \mathrm{~V} / \mathrm{ft}^{2}$ (as shown above in the section 3.1.), an array of $\mathrm{EH} 2 \mathrm{Ns}$ as large as the full area of the LED compartment ( $2 \mathrm{ft} \times 1.5 \mathrm{ft}$ ) would then be able to light up all of the LEDs using the proposed $\mathrm{SiC}$ mechanism. On top of the plexiglass, solar panels were placed with wheels riding along a track to simulate the car with the solar panel on its bottom.

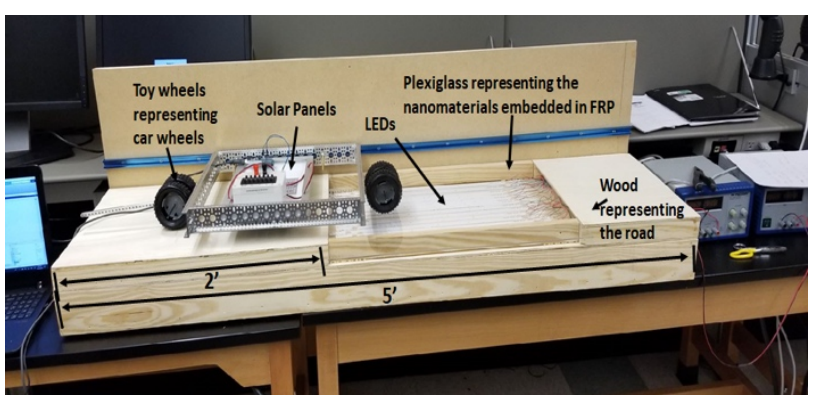

Fig. 6. Lab-scale proof-of-concept prototype.

\subsubsection{Test results}

Case 1: Stationary Test: For the purpose of verifying the proposed concept of $\mathrm{SiC}$, the LEDs were first powered using a DC power supply. The output power of the stationary 10-watt Eco-Worthy solar panel without plexiglass was measured and presented in the IV curve depicted in Figure 7. The maximum output power was measured to be $7.5 \mathrm{~W}$. The lumens per watt $(l u x)$ were also measured and it came out to be 120.1 lux with the plexiglass and 163.6 lux without it.

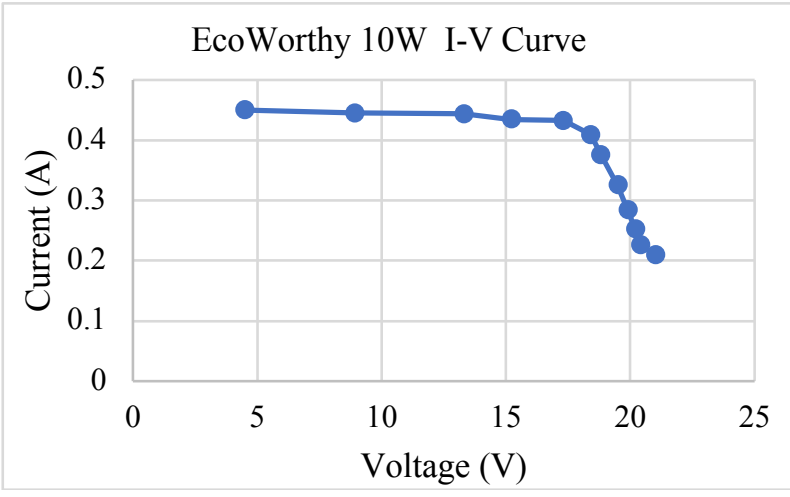

Fig. 7. Electrical characteristic of the EcoWorthy $10 \mathrm{~W}$ solar panel lighted from LEDs.

Case 2: In-motion Test: In this test, the solar panel was moved along the track with a velocity of $1 \mathrm{ft} / \mathrm{sec}$ to simulate the car with the solar panel on its bottom as shown in Figure 6. Figure 8 depicts the output voltage, current and power measured while the simulated vehicle is in motion. The maximum output power was $2.6 \mathrm{~W}$ from one panel with a load of $40 \Omega$ tracking the maximum power point. The prototype has three panels to cover all the LEDs area and therefore the maximum output power for the prototype is $7.8 \mathrm{~W}$. 


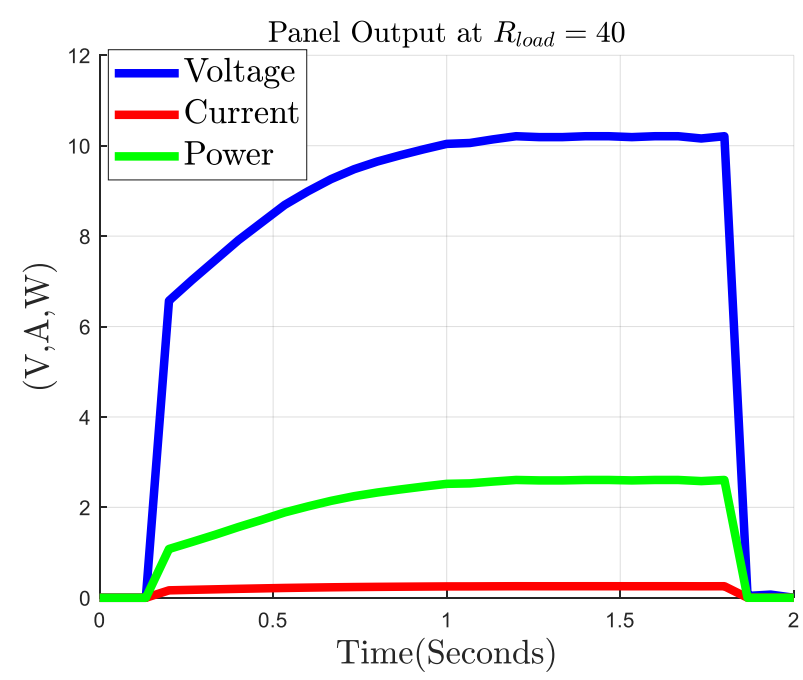

Fig. 8. Output voltage, current and power measured while simulated vehicle in motion.

\section{Conclusions}

The paper presented a novel smart charging system for future electric vehicles - namely, smart illuminative charging ( $\mathrm{SiC}$ ). It is a novel (induction coil-free) wireless charging mechanism that uses nanomaterialspowered LEDs embedded within the road pavement and thin film solar panels placed on the bottom of the electric vehicles. Light mediates energy transfer between LEDs and solar panels. To better protect the LEDs from the heavy vehicle load whilst still harnessing their electricto-optical energy conversion capability, certain flexible polymer overlay materials will need to be developed and placed onto the pavement surface. The paper presented the initial, albeit promising, results on the great promise of the nanomaterials to harvest energy and provide electricity to light up the LEDs. A lab-scale proof-ofconcept prototype was also demonstrated to verify the system capability.

\section{References}

1. Gerssen-Gondelach, S.J. and Faaij, A.P.C. (2012). Performance of batteries for electric vehicles on short and longer term. J. Power Sour. 212, 111.

2. Etacheri, V., Marom, R., Elazari, R., Salitra, G., and Aurbach, D. (2011). Challenges in the development of advanced Li-ion batteries: A review. Energy Environ. Sci. 4, 3243.

3. S. Manzeli et al. (2017). 2D transition metal dichalcogenides. Nature Reviews Materials 2, 17033.

4. B. Radisavljevic et al. (2011). A single-layer MoS2 transistors, Nature Nanotechnology. 6, 147.

5. A. Splendiani et al. (2010). Emerging photoluminescence in monolayer MoS2, Nano Letters. 10, 1271.

6. S.Z. Butler et al. (2013). Progress, challenges, and opportunities in two-dimensional materials beyond graphene. ACS Nano 7, 2898.
7. W. Wu et al. (2014). Piezoelectricity of singleatomic-layer MoS2 for energy conversion and piezotronics. Nature. 514, 470.

8. H. Zhu, et al. (2015). Observation of piezoelectricity in free-standing monolayer MoS2. Nature Nanotechnology. 10, 151.

9. E. Okogbue et al. (2018). Centimeter-Scale Periodically Corrugated Few-Layer 2D MoS2 with Tensile Stretch-Driven Tunable Multifunctionalities. ACS Applied Materials and Interfaces. 10, 30623.

10. A.C. Ferrari et al. (2006). Raman Spectrum of Graphene and Graphene Layers. Phys. Rev. Lett. 97, 187401. 